\title{
CORRELAÇÃO ENTRE A AUTOMEDICAÇÃO DE CORTICOIDES COM O DESENVOLVIMENTO DE RESISTÊNCIA À INSULINA E COMO O FARMACÊUTICO PODE AJUDAR NA PREVENÇÃO
}

\author{
Amanda Monsores da Conceição ${ }^{1}$ \\ Thiago Pereira de Abreu ${ }^{2}$
}

RESUMO: Sintetizados pela glândula suprarenal, os hormônios esteróides são chamados de glicocorticoides (GC) ou corticosteroides. Hoje, trabalha-se a certeza de que a fabricação destes hormônios pela indústria farmacêutica, obtém sucesso em diversos tratamentos, entretanto, com a cultura da automedicação os corticoides tornaram-se um vício e consequentemente um risco gravíssimo para a população que faz o uso indevido. A busca pelo tratamento com corticoides, na maioria dos casos é relacionada a sua ação antiinflamatória e na terapia de doenças autoimunes, ademais, o uso de corticoides pode levar ao surgimento de diabetes se utilizados por um longo período. O profissional farmacêutico, uma vez tendo conhecimento de tais perigos, ainda que tenha a prescrição médica em mãos deve fazer os devidos esclarecimentos e questionamentos ao paciente no ato da dispensação, a fim de promover a prevenção da doença e reafirmar a orientação correta relativa ao uso de corticoides e consequentemente, evitar que o paciente faça uso indevido ou prolongado do medicamento após o período em que lhe foi prescrito.

Palavras-chave: Corticóides. Suprarenal. Automedicação. Farmacêutico.

ABSTRACT: Synthesized by the adrenal gland, steroid hormones are called glucocorticoids or corticosteroids. Nowadays, though, the certainty is that the production of these hormones by the pharmaceutical industry is successful in several treatments. However, with a culture of self-medication, corticosteroids have become an addiction and, consequently, very dangerous for the population that doesn't take them properly. The looking for treatment with corticosteroids, in most cases, are related to it's antiinflammatory action and as therapy for autoimmune diseases, but the use of corticosteroids can lead to the onset of diabetes if used for a long period. The pharmacist professional, once aware of such dangers, even with the medical prescription in hand, must make the necessary clarifications and questions to the patient at the time of dispensing, to promote prevention of diseases and reaffirm the correct orientation regarding the use of corticosteroids and, consequently, prevent the patient from taking the medication improperly or for longer than the period in which it was prescribed.

\footnotetext{
I Graduação em farmácia pela UNIG - Universidade Iguaçu-Faculdade de Ciências Biológicas e da Saúde. E-mail: amandamonsores3o@gmail.com.

${ }^{2}$ Professor e orientador pela UNIG - Universidade Iguaçu Faculdade de Ciências Biológicas e da Saúde.
} 
Keyword: Corticosteroids. Adrenal gland. Automedication. Pharmacist.

\section{INTRODUÇÃO}

Os glicocorticóides são hormônios esteróides, sintetizados no córtex da glândula adrenal, que afetam o metabolismo dos carboidratos e reduzem a resposta inflamatória (GOODMAN \& GILMAN, 2003).

A busca pelo tratamento com corticóides, na maioria dos casos é relacionada a sua ação antiinflamatória e na terapia de doenças autoimunes, ademais, o uso de corticóides pode levar ao surgimento de diabetes se utilizados por um longo período de tempo.

Tendo em vista o acúmulo de açúcar decorrente do uso prolongado ou indevido do hormônio, deve-se levantar os seguintes questionamentos antes de iniciar a terapia com glicocorticóides: o paciente tem resistência insulínica? Existe a necessidade do uso por tempo prolongado? $O$ paciente foi questionado sobre um possível favorecimento ao desenvolvimento de diabetes pela terapêutica a iniciar-se com corticóide?

O profissional farmacêutico, uma vez tendo conhecimento de tais perigos, ainda que 646

tenha a prescrição médica em mãos deve fazer os devidos esclarecimentos e questionamentos ao paciente no ato da dispensação, a fim de promover a prevenção da doença e reafirmar a orientação correta relativa ao uso de corticóides e conseqüentemente, evitar que o paciente faça uso indevido ou prolongado do medicamento após o período em que lhe foi prescrito.

\section{OBJETIVO GERAL}

O presente estudo traz informações sobre corticoides, os efeitos colaterais e o perigo do uso sem a orientação correta.

Enaltecer a importância da atenção farmacêutica, na anticoncepção de doenças e efeitos colaterais relacionados a automedicação com corticoides, dentre eles a resistência à insulina adquirida por mal uso.

Advertir sobre o perigo da automedicação de corticoides e como o uso indevido pode acarretar o desenvolvimento da resistência à insulina. 


\section{OBJETIVOS ESPECÍFICOS}

Argumentar sobre o perigo da automedicação com corticoide Acautelar sobre o uso indevido e o possível desenvolvimento de resistência à insulina

A importância da atenção farmacêutica na precaução de tais efeitos indesejados causados pelo uso de corticoides.

Acautelar sobre o uso indevido e o possível desenvolvimento de resistência insulina.

\section{JUSTIFICATIVA}

como parte fundamental da pesquisa, baseei-me nas pesquisas que atinam para os perigos do uso indevido dos medicamentos, trazendo mais uma vez a tona o perigo da automedicação, e a importância da orientação com o profissional farmacêutico antes do início de terapias com corticoides, mesmo tendo a orientação médica. a pesquisa traz também, informações por muitos desconhecidas como o possível desenvolvimento de diabetes pelo uso de corticóides.

\section{METODOLOGIA}

Foram feitas leituras e pesquisas detalhadas através de artigos científicos, livros e revistas científicas a fim de trazer ao conhecimento a produtividade dos hormônios em nosso organismo, o melhor horário para a terapêutica com o medicamento corticoide, suas funções benéficas e os malefícios do seu uso indevido ou incorreto quando sem a orientação correta do médico e do profissional farmacêutico.

Foi feita uma pesquisa de campo para descobrir o corticoide comumente utilizado, em terapêutica com corticoides, se foram prescritos por médicos, se não, como descobriram sua utilidade, para qual finalidade fizeram uso de corticoides, se sabem do risco do desenvolvimento de diabetes através da auto medicação com corticóides.

\section{FARMACOLOGIA DOS GLICOCORTICÓIDES}

Denominadas suprarrenais ou adrenais, as glândulas encontradas acima de cada um dos rins, são divididas em duas partes: córtex (parte externa da adrenal) e medula (interior da glândula adrenal). 
$\mathrm{Na}$ parte interior da adrenal, ocorre a síntese dos hormônios do sistema nervoso, são eles:

Epinefrina (adrenalina) que é liberado sempre que o corpo passa por situação de demasiada emoção ou grande estresse, acionando em nós uma espécie de reação de defesa.

Norepinefrina (noradrenalina) que envolve a concentração e ações de resposta no cérebro, durante o dia noradrenalina nos deixa atentos e ao anoitecer tem uma diminuição na sua atividade.

Os hormônios desenvolvidos no córtex da suprarrenal, derivam-se do colesterol, e podem ser divididos em grupos como:

Figura I - Fórmula da epinefrina

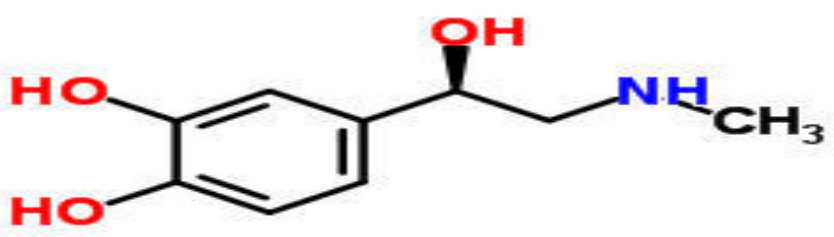

Fonte: Berecek KH, Brody MJ (1982)

Figura 2 - Fórmula da norepinefrina

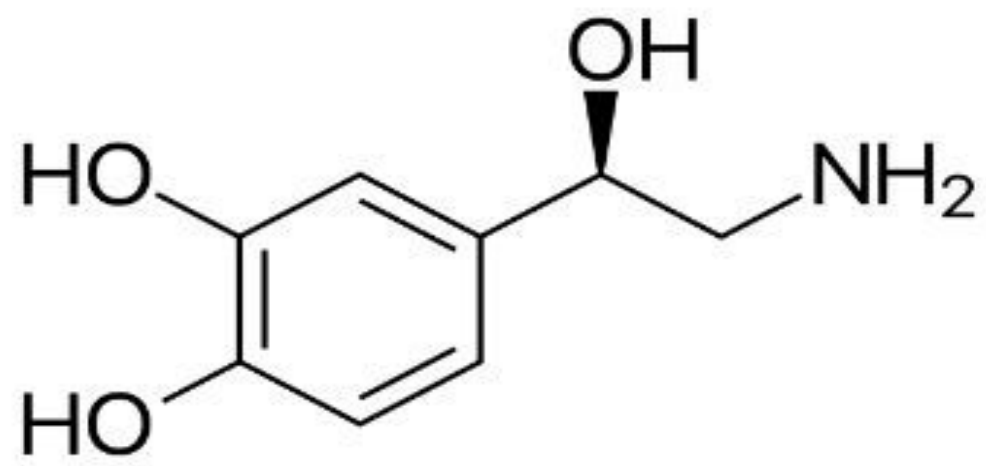

Fonte: Berecek KH, Brody MJ (1982) 
Mineralocorticóides (atuam no equilíbrio de sódio e $\mathrm{H}_{2} \mathrm{O}$ no organismo), glicocorticoides (tem ação anti-inflamatória e imunossupressora) e andrógeno (hormônios sexuais), a discriminação de cada um se deve a enzima que atuará sobre ele, enzimas estas encontradas no córtex da glândula adrenal.

Figura I - Córtex

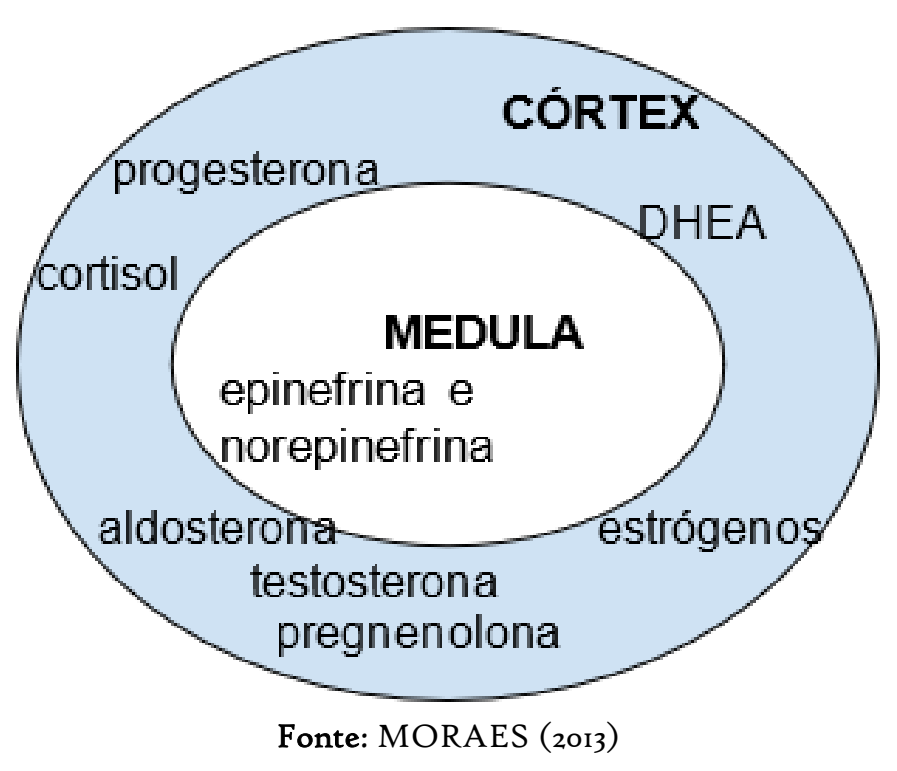

Mesmo sendo hormônios produzidos naturalmente pelo corpo humano, glicocorticoides (GC) também são sintetizados por indústrias farmacêuticas, com finalidade de uso anti-inflamatório, imunossupressor (nas doenças autoimunes) e no tratamento de algumas alergias. O corticoide sintético é utilizado em doses bem maiores do que as produzidas diariamente pelo nosso organismo, por isso são muito mais eficazes na ação imunossupressora e anti-inflamatória. Em outras palavras, quanto maior a dose, maior o efeito anti-inflamatório, em contrapartida, maior o efeito dos GC no metabolismo de carboidratos, ou seja, eles aumentam a degradação de proteínas, trazendo como resultado desta degradação mais glicose para o corpo e consequentemente elevam a produção e o acúmulo de açúcar no sangue. Naturalmente, os hormônios produzidos pelo nosso organismo, já tem a função de elevar os níveis de açúcar, notoriamente, o uso dos corticoides produzidos pela indústria farmacêutica aumenta ainda mais esses níveis. daí, justifica-se o excesso de glicose, o seu acúmulo e o desenvolvimento da diabetes. 


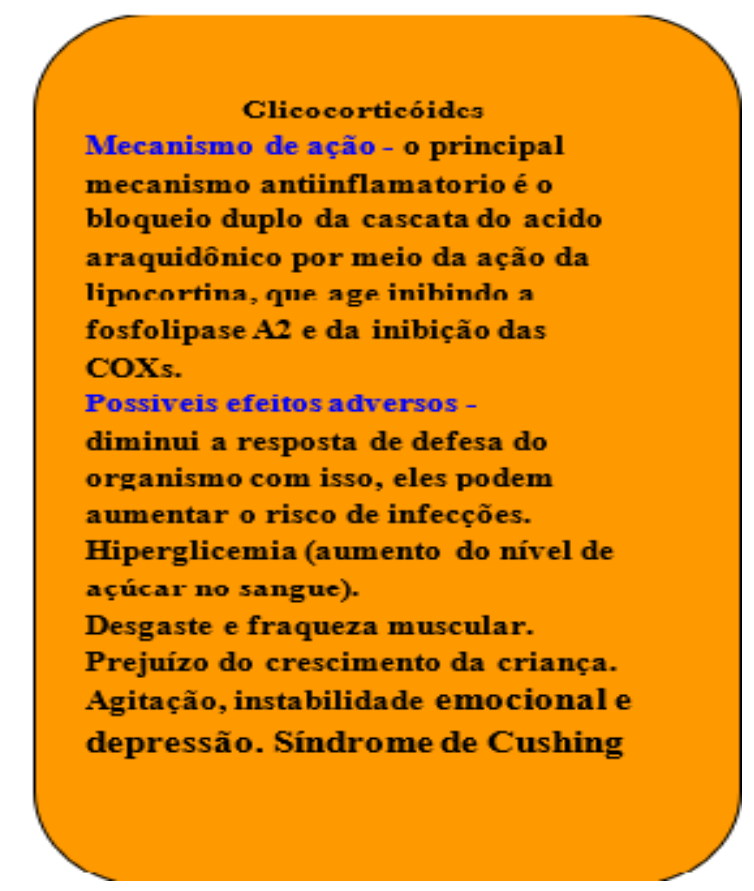

\section{I. Associando o natural e o sintético}

Sintetizados pela glândula suprarrenal, os hormônios esteroides são chamados de glicocorticoides (GC) ou corticosteroides. hoje, trabalha-se a certeza de que a fabricação destes hormônios pela indústria farmacêutica, obtém sucesso em diversos tratamentos, entretanto, com a cultura da automedicação os corticoides tornaram-se um vício e consequentemente um risco gravíssimo para a população que faz o uso indevido.

Levando em consideração que são estes, hormônios produzidos pelo organismo, o organismo que recebe doses contínuas e a longo prazo de tais hormônios sintéticos, tende a parar de produzir os mesmos, sendo obrigatório um desmame para a descontinuidade do tratamento, a fim de evitar efeitos colaterais severos pela falta dos glicocorticoides que são essenciais para o bom funcionamento do organismo.

O que difere o natural do sintético é sua estrutura química, uma vez que o hormônio natural, produzido pelo organismo, tem em seu DNA vestígio do seu precursor o colesterol. mas a ação tanto de um quanto de outro tem a mesma finalidade, claramente evidenciando que o sintético com que se mantém entre 5-1o mg se mantém dentro da dose produzida pelo organismo, e nesta dosagem, sua ação geralmente tem finalidade antiinflamatória. sendo assim, distante o risco de efeitos colaterais, o risco de efeitos colaterais é maior para quem faz uso de doses altas e por longo período de tempo. 
A produção normal do organismo sofre alterações ao longo do dia, tendo sua alta concentração ao amanhecer e seu nível diminuído no fim da noite. Quando se está para iniciar a terapêutica com corticoides, o ideal é que se faça uso matinal, pois se assemelha com a produção normal do organismo, já que pela manhã a produção dos hormônios encontra-se em seu ponto alto.

entre tanto, ao findar um tratamento deve-se acautelar sobre o risco do organismo ter diminuído ou parado de produzir o hormônio, tendo em vista que ele recebia uma dose diária do hormônio durante a terapia, o organismo pode entender que não existe a necessidade de produzir o hormônio, neste caso dada a importância do hormônio para o organismo humano, é necessário um término gradativo do tratamento. o famoso desmame, para que o corpo volte a produzir o hormônio como de costume e não haja desconforto algum, após o término do tratamento.

\subsection{Degradação de carboidrato e o acúmulo de açúcar no sangue}

Os glicocorticoides sintéticos têm ação idêntica aos hormônios naturais do organismo,com isso, temos um resultado de mais metabolização de carboidratos, tendo consequentemente, maior concentração de açúcar no sangue.

A metabolização do carboidrato eleva a gliconeogênese hepática, o GC também é antagonista periférico de insulina, dificultando a captação de glicose no músculo e tecido gorduroso, induzindo assim, a hiperglicemia.

\section{carboidratos}

diminui a utilização periférica da glicose

diminui a ação da insulina

aumenta a neoglicogenese ( produção de glicose)

Visto isto, os corticoides podem desta maneira desencadear o desenvolvimento de diabetes em pessoas pré-dispostas ou não, pode também, interferir no tratamento dos pacientes diagnosticados com a doença. fazendo necessário um cuidado especial à pacientes hiperglicêmicos que estejam prestes a iniciar a terapêutica com glicocorticoides. 


\subsection{Associação de corticóides e medicamentos antidiabéticos}

Pacientes pertencentes ao grupo de pessoas hiperglicêmicas, tem sua terapia medicamentosa, com finalidade de tentar controlar a hiperglicemia ameaçada ao dar início a terapêutica com corticosteroides, uma vez que os GC têm resultado hiperglicêmico no organismo, o profissional de saúde precisa inspecionar o paciente quanto a terapia medicamentosa utilizada pelo paciente em pró do tratamento da diabetes e aumentar a dose de seus antidiabéticos casa seja necessário.

A concentração de glicose no sangue aumentada traz riscos vitais para pacientes com hiperglicemia, a orientação farmacêutica sobre uso do medicamento, tempo correspondente e horário é fundamental, para o tratamento seguro, segundo a prescrição médica.

Afirma-se mais uma vez a importância de um atendimento profissional antes de fazer uso de medicamentos, é necessário ter conhecimento da patologia do paciente, para que sejam eliminados ou amenizados os riscos vitais que o medicamento pode trazer durante a terapia.

\section{DADOS DA PESQUISA}

Foi realizada uma pesquisa a fim de afirmar a falta de conhecimento dos riscos do tratamento com corticosteroides em pacientes hiperglicêmicos, pacientes com predisposição à doença e pacientes sem ligação alguma com a doença. segundo dados da pesquisa realizada entre os dias 23 e 29 do mês de agosto do ano 202I deu-se os seguintes resultados:

Figura 2 - Resultados da pesquisa I

você é diabético?

71 respostas
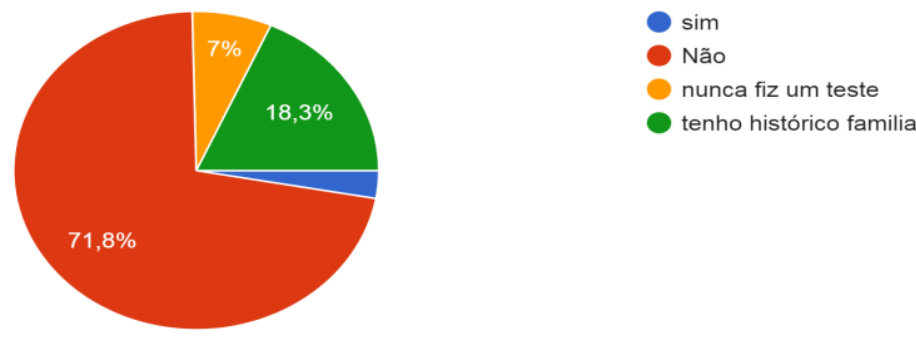
Figura 3 - Resultados da pesquisa 2

procura o medico com frequência?

70 respostas

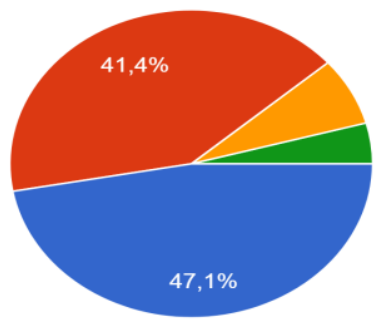

sim, sempre que tem algo fora do normal comigo.

somente em ultimo caso, eu tento resolver em casa mesmo.

eu sempre vou a farmácia e falo com o rapaz do balcão.

- eu sempre repito os tratamentos anteriores, sem voltar ao medico.

Figura 4 - Resultados da pesquisa 3

você já fez uso de algum destes medicamentos?

71 respostas

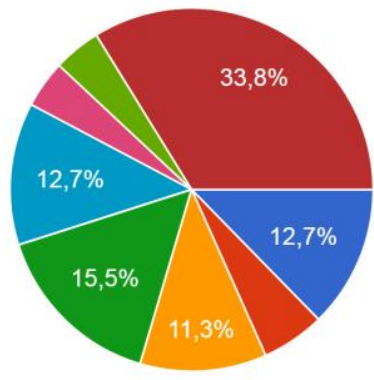
injeção de diprospan
injeção de beta trinta
Decadron / dexametasona (comprimidos ou líquidos)
predsin (comprimidos ou liquido)
prelone ( comprimidos ou liquido)
prednisolona liquido ou comprimidos)
corticorten
mudoral, mud creme ou triancinolona
nenhum

Figura 5 - Resultados da pesquisa 4

para qual finalidade fez uso de corticoides?

71 respostas
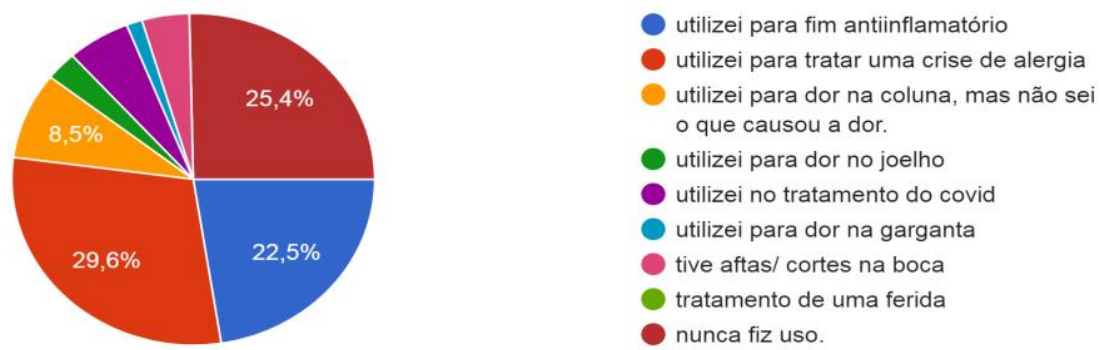
Figura 6 - Resultados da pesquisa 5

como conheceu este medicamento?

71 respostas

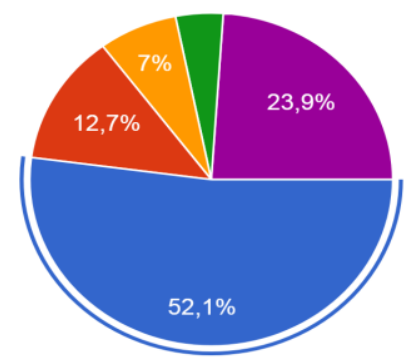
foi prescrito pelo medico
alguém me disse que era bom para o meu caso
pesquisei na internet
o medico prescreveu para alguém da
minha família e eu lembrei o nome
não conheço nenhum.

Figura 7 - Resultados da pesquisa 7

com que frequência faz uso de corticoides?

71 respostas

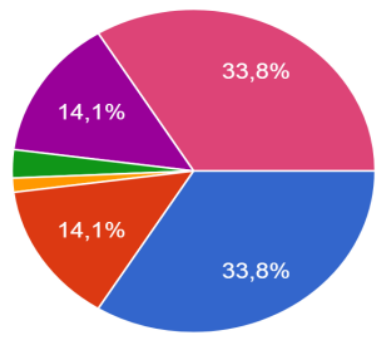

usei apensa quando o medico prescreveu

sempre que tenho crise, nunca voltei ao medico depois que descobri este tratamento

Figura 8 - Resultados da pesquisa 8

você já ouviu ou leu a respeito dos efeitos colaterais dos corticoides, dentre eles o desenvolvimento de diabetes?

71 respostas

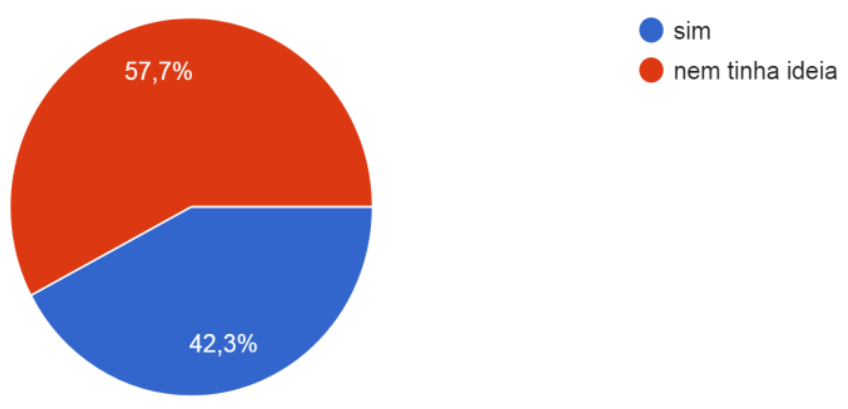


Figura 9 - Resultados da pesquisa 9

alguma vez leu ou ouviu dizer que o uso do corticoide pode inibir ou cortar o efeito dos medicamentos que tratam a diabetes?

71 respostas

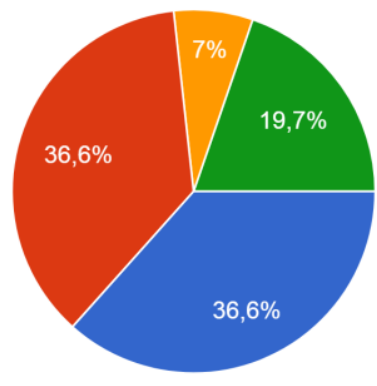

sim fui orientado pelo medico a fazer uso somente pelo tempo determinado por ele.

não, ninguém me orientou por isso faço uso sempre que acho necessário.

não sabia, e eu sou diabético.

não sabia, e eu usei porque todo mundo tomou.

\section{DISCUSSÃO}

A partir da pesquisa citada anteriormente, podemos afirmar que ainda falta muita informação sobre o risco de diabetes mediante o uso de corticoides sem a orientação correta, ainda existem indivíduos que por falta de conhecimento dos riscos automedicam-se com corticoides e desconhecem o risco do desenvolvimento de diabetes. O número de pessoas desinformadas e que praticam a automedicação ainda é preocupante segundo a pesquisa.

Reforça-se a importância de orientações profissionais, a automedicação pode levar aos efeitos indesejados de medicamentos, vale lembrar que o que difere o medicamento do veneno é a dose! e é muito importante a orientação profissional antes de iniciar-se qualquer terapêutica medicamentosa, respeitando a orientação profissional médica e também a orientação do profissional farmacêutico.

Ainda relativo a pesquisa, é gratificante afirmar que grande parte dos colaboradores da pesquisa, tem o bom hábito de procurar um auxílio profissional (médico) toda vez que uma enfermidade os assola, trazendo para si, mais segurança e eficácia dos tratamentos feitos com corticosteróides ou não. 


\section{CONCLUSÃO}

A busca pelo tratamento com corticoides, na maioria dos casos é relacionada a sua ação anti-inflamatória e na terapia de doenças autoimunes, ademais, o uso de corticoides pode levar ao surgimento de diabetes se utilizados por um longo período.

Por isso, é importante respeitar a terapia prescrita, não repetir por conta própria caso os sintomas reapareçam, neste caso, o correto é retornar ao médico.

Vale lembrar a importância de orientar-se com o profissional farmacêutico no ato da compra para sanar qualquer dúvida ainda restante sobre tal mediação, e nunca se automedicar por indicação de terceiros.

\section{BIBLIOGRAFIA}

Departamento de Gestão e Incorporação de Tecnologias e Inovação em Saúde DGITIS/SCTIE e Centro Colaborador do SUS: Avaliação de Tecnologias e Excelência em Saúde - CCATES. Rio de Janeiro, 2020. Disponível em: <http://antigo.saude.gov.br/images/pdf/2020/September/o3/NotaTecnica-

Dexametasona-Covidig-Agosto2020.pdf $>$. Acesso em: 16 set. 202I.

DAMIANI, Durval. Corticoterapia e suas repercussões: a relação custo-benefício. Rio de Janeiro, 2020. Disponível em: <https://www.researchgate.net/profile/Thais-Manna2/publication/2381006I8_Corticoterapia_e_suas_repercussoes_a_relacao_custobeneficio_Repercussions_of_corticotherapy_the_costbenefit_ratio_Repercuciones_de_la_corticoterapia_la_relacion_costobeneficio/links/54ceseafocf29ca8rofbf5ab/Corticoterapia-e-suas-repercussoes-a-relacaocusto-beneficio-Repercussions-of-corticotherapy-the-cost-benefit-ratio-Repercuciones-dela-corticoterapia-la-relacion-costo-beneficio.pdf $>$. Acesso em: I6 set. 202I.

SANARMED. Corticoides. Rio de Janeiro, 2020. Disponível em: 〈https://www.sanarmed.com/resumo-corticoides〉. Acesso em: I6 set. 202I.

NUNES, Dejair. REAÇÕES ADVERSAS RELACIONADAS AO USO DE CORTICOIDES EM IDOSOS: REVISÃO DE LITERATURA. Bahia, 2or6. Disponível em: 〈http://famamportal.com.br:8082/jspui/handle/r23456789/266>. Acesso em: I6 set. 2021. 
UFMG. Cuidados em relação ao uso de medicamentos a base de corticoides. Minas Gerais, 2021. Disponível em: <https://www.medicina.ufmg.br/wpcontent/uploads/sites/75/2015/o8/Cartilha-Corticoide-final.pdf $>$. Acesso em: I6 set. 2021.

Pesquisa: https://docs.google.com/forms/d/ItmNf_MJA7YxqfLYaVEZMohUAzTQwaiiCsphFUPEtfk/edit 